Memorandum No. 1746

Knowledge graph analysis of particles in Japanese

C. Hoede

January, 2005 


\title{
Knowledge Graph Analysis of Particles in Japanese
}

\author{
C. Hoede \\ Faculty of Electrical Engineering, Mathematics and Computer Science \\ University of Twente \\ P.O.Box 217 \\ 7500 AE Enschede, The Netherlands
}

January 20, 2005

\begin{abstract}
The theory of knowledge graphs is a structuralistic theory of language. Its ontology consists of eight types of binary relationships and four types of so-called frames. The relationships connect so-called tokens, that represent semantic units. In this way a graph structure arises.

Japanese particles are investigated against the background of knowledge graph ontology. It is shown that the structure of Japanese closely resembles the structure of the knowledge graph representation of language.
\end{abstract}

Key words: Knowledge graphs, Japanese, particles, structuralistic.

AMS Subject Classifications: 05C99, 68F99.

\section{Introduction}

An important example of a structuralistic theory of language was developed by Ebeling around 1978. The author's attention to that theory was drawn by Kortlandt, who made available a summary, edited by Wiedenhof and Smeets [5]. In a comparative study it turned out that Ebeling's theory and the theory of knowledge graphs, developed from 1982 on, have great similarity [7].

Both theories intend to be universal in the sense that a representational system is proposed, that should be able to represent any natural language. The main difference between the two theories is that the relationships between linguistic units in knowledge graph theory are completely semantic in nature. The types of relationships are considered to reflect the way neural networks process impressions. As such they are meta-linguistic descriptions on the sub-word level. A thought is seen as a specific configuration of awareness units, the tokens, linked by basic types of relationships, the ontology of knowledge graph theory. Thus the thought is a labeled graph, in which no words occur. All people are assumed to 
have the same graph "in mind" for given impressions.

The differences between languages come in when the "framing and naming" process takes place. Certain subgraphs of the graph representing a thought are seen as a unit, a frame, and are "baptized" with a word. The way this is done differs from language to language. We can assume a dog is seen the same way by an English speaking person as by a French speaking person. That the word "chien" is used by the second one exemplifies our point.

However, suppose that a graph representing a thought, and therefore called subgraph of the mind graph a person has in mind, is to be "brought under words". It are not the verbs and nouns, the complex structures, that pose a challenge, but rather those words that express the glue between them. These words, like e.g. prepositions, express the basic semantic relationships between the main categories of words. We will give some more information, in the next section, about the way this is done in knowledge graph theory.

Having finished the comparison of the two structuralistic theories, Kortlandt invited the author to have a look at the publications available on his website. The paper that attracted my attention was titled "Japanese WA,MO,GA,WO,NA,NO". These little words are called particles. This paper will also focus on Japanese particles. What made things exciting was the discovery that Japanese is, in a sense, the perfect language from the point of view of knowledge graph theory!

Much of what will be said is based on findings in the Handbook of Japanese Grammar by H.G. Henderson [6]. The structure of that book is remarkable. Of 360 pages 320 pages are dedicated to a dictionary of particles, suffixes and other connectives. Connecting linguistic units is what Japanese is all about. But that is precisely the paradigm of knowledge graph theory!

\section{A short survey of knowledge graph theory and re- sults}

For graph theoretical terminology the reader is referred to any of the many books on graph theory, e.g. that of Bondy and Murty [3]. The tokens, representing awareness units, are the vertices of the graph. The relationships between them are the labeled arcs, oriented, and edges, non-oriented. The choice of the labels, the types of relationships supposed to be recognized by the brain, is defended as follows.

The granular structure of the world leads to the recognition of sets. Two sets A and B can have four relationships; equality, $A=B$, leading to an edge of type EQU; inclusion, $A \subset B$, leading to an arc of type SUB; intersection, $A \cap B \neq \emptyset$, leading to an edge of type ALI; non-intersection, $A \cap B=\emptyset$, leading to an edge of type DIS. SUB refers to the concept of SUBset, ALI to ALIkeness and DIS to DISparateness. 
The space-time aspect of the world leads to the recognition of ordering. Therefore an, oriented, ORD-relationship is introduced. Also an asymmetric CAU-relationship is taken up in the ontology to represent causal relationships. The CAU-link is quite important in modeling the world, although Hume rightly argued that it is a composite type of link, the ORD-link being more basic, see e.g. Russell [13].

Finally there are two types that may be seen as stemming from the fact that there are other minds that one can perceive in the world. These two types are the PAR-relationship and the SKO-relationship. The first one models attribution that makes one something a PARt of another something, but in the mind only. "This is a nice pitbull" is a statement that not necessarily is held to be true by everybody. "Nice" is attributed to "pitbull" by the speaker so $\stackrel{\text { nice }}{\longrightarrow} \stackrel{\text { PAR }}{\longrightarrow}$ pitbull would be the graph representation. We will come back to merological relationships later. SKOlem is the name of a logician. The SKO-link, that like the PAR-link is oriented, represents informational dependency, as occurs in the concept of mapping in mathematics.

Next to these eight types of relationships, there are four types of frames and therewith four types of frame relationships. The most basic frame is that representing the subgraph of the mind graph that is focused upon. The subgraph is framed and seen as a unit that is represented by a token, vertex, again. The token can now be named, i.e., a word can be attached to it. This is where words come into the theory. The "framing and naming" process can be different for different languages. In fact, within a certain language the process can be different for different users! Here is the place to mention one of the basic assumptions of the theory: The structure is the meaning. The meaning of a word is defined as the subgraph that is framed and named by that word.

The representation of a naming is either

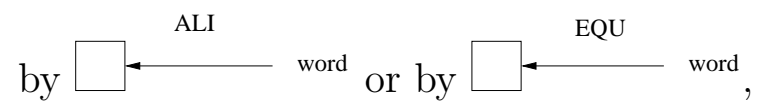

so by oriented ALI- or EQU-links. The first type is used between a token and a word, when the word is typing the token in the context of a taxonomy or type-hierarchy. The top-element is the word "something", the type of any token :

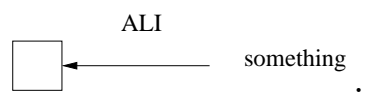

Note that "token" is used on the meta-level, whereas "something" is used to talk about the token in English. The oriented EQU-link is used for instantiation. "Elephant Jumbo" is represented as

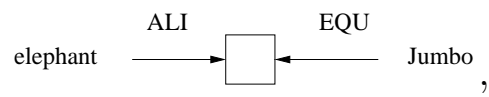


read as "something" with type (alike an) elephant with instantiation (being equal to) "Jumbo".

The frame with label BE may be seen as expressing the auxiliary verb "be". The contents of a frame are in a relationship with the name attached to the frame. In most cases a word graph, there should be one for every word, contains other words, occurring in the definition of the word, i.e., belonging to its meaning, which is that word graph! This puts the occurring word in an FPAR-relationship (Frame PARt-relationship). Within the token for "elephant" the concept "trunk" may occur, if a trunk is seen as element of the definition. The trunk is then seen as a property of an elephant : trunk $\stackrel{\text { FPAR }}{\longrightarrow}$ elephant. If it is seen as a part we would represent this by trunk $\stackrel{\text { SUB }}{\longrightarrow}$ elephant. We already mentioned that

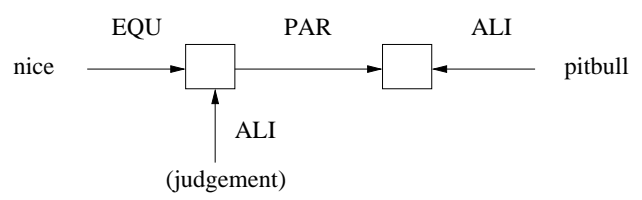

expresses that nice is an attribute of pitbull. So knowledge graph theory distinguishes three merological relationships. In English we say "property of", "part of" and "attribute of" respectively "with property", "with part" and "with attribute". This brings us closer to our main theme. The words "of" and "with" should have word graphs too. We will come back to them in Section 3.

The history of the theory is as follows. First only three types of relationships, including in particular the CAU-link, were considered in the attempt to extract expert systems from texts. This led to the theses of Bakker [1], de Vries [15] and Smit [14].

Then the goal was extended and it was investigated how the representational formalism had to be extended to represent all of natural language and logic. Willems' thesis [16] was titled : The Chemistry of Language, whereas van den Berg[2] showed that by introducing four types of frames all logical systems could be represented. For this a NEG-frame, for NEGation, a POS-frame, for POSsibility and a NEC-frame, for NECessity, had to be introduced. The basic frame can be seen as representing units together, so may be named AND-frame. Together with the NEG-frame we have a functionally complete system of logical connectives. The two other types of frames are needed to represent modal logics. We will not go deeper into this. Let me only mention that in our theory we are inclined to state that thinking is linking somethings.

Van den Berg and Willems introduced the SKO-arc in order to represent universal quantification in logic. A SKO-loop on a token expresses that the token is informationally dependent on itself alone, and may therefore be anything, within the given restrictions. This is the seen as expressing "for all". However, the SKO-link may be used in another way as well, closer to linguistic theory. Let us consider the representation of verbs. We consider the sentence "dog bite(s) man", that is represented as : 


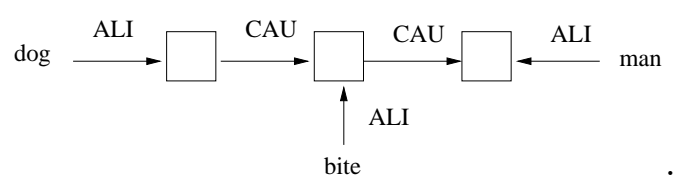

Something of type bite (is) cause(d) by something of type dog and caus(ing) (having effect on) something of type man, may be a reading of this sentence graph. Any reading, uttering, of this sentence graph must be in terms of words that are identical with, or at least cover, certain subgraphs of it. The way this can be done depends on the grammar of the language, on the way the word types may be linked. "Dog" and "man" are nouns, whereas "bite" is a verb but, apart from the time aspect, there is not much difference between them. The ORD-aspect of the CAU-link is related to ordering in time, inherent in the verb word graph. A verb "asks for" a subject and an object, unless the verb is intransitive. In a causation

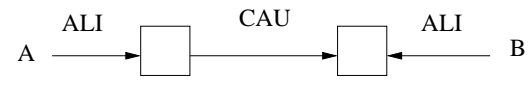

the independent variable is A. B is "informationally dependent on" A. If we only want to express the syntactical relationship between nouns and verbs we may choose the SKO-link as a representation. The considered sentence then has the structure :

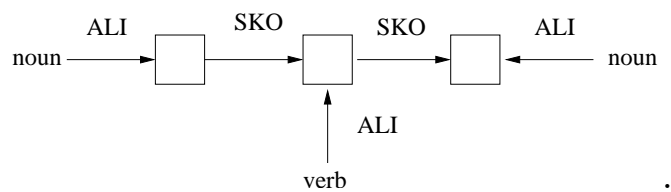

We can make a clear distinction between semantic word graphs, expressing the meaning of words and syntactic word graphs, expressing the "functioning" of words. Note that syntactic word graphs can be seen as semantic word graphs for word types, see e.g. Zhang [17]. Another clear distinction can be made between "agent" and "patient" on the one hand, with word graphs like

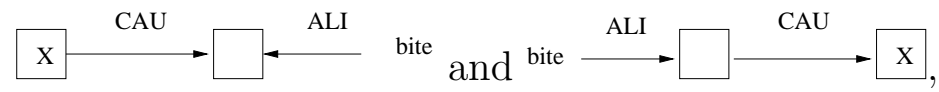

and "subject" and "object" on the other hand, with word graphs

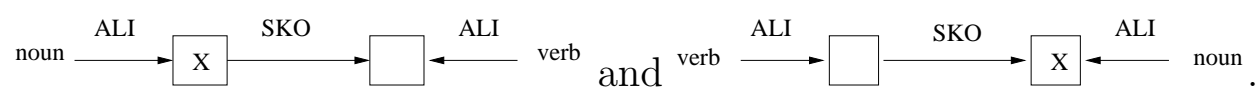

The crosses indicate that the focus is on the specific token.

Word graphs have now been studied for three sets. Hoede and Li [8] studied the first set, consisting of verbs, nouns and prepositions. The potential of the theory came forward in the study of Chinese words for "in". In English the word graph 


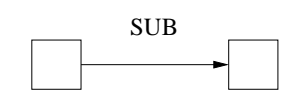

would be given. However, Chinese knows 15 different words for "in", depending on the context. Indeed, 15 different word graphs could be indicated grasping the different contexts. Hoede and Liu [9] studied the second set, consisting of adverbs, adjectives and Chinese classifiers. Because of their common features these three classes of words were together called adwords, next to verbs and nouns the third major class of words. Hoede and Zhang [10] studied the third set of words, the so-called logic words, as used in English and Chinese. An important example of a logic word is the word "all".

Historically it is important to mention that the idea of putting a frame around two or more tokens to represent the word "and" was brought forward already in 1885 by the American philosopher Peirce [12].

\section{The glue of language}

The picture developed in knowledge graph theory sofar is that of basic linguistic units like verbs, nouns and adwords, being linked by other words. As the representation is by means of vertices and labeled arcs and edges, where the vertices represent mainly the verbs, nouns and adwords, the other words, that "glue" these main classes together, must have word graphs that have the labelled arcs and edges as main constituents, whereas for the logic words we expect frames to play an essential role too. This is indeed the situation and our discussion of Japanese particles will confirm this in a striking way.

We will start by considering the ORD-link. The total graph of this link has a vertex for the arc as well and unlabelled arcs for expressing the orientation:

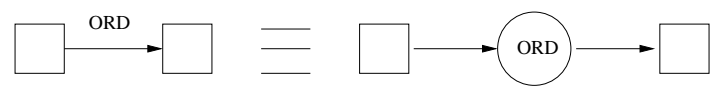

The total graph has two subgraphs :

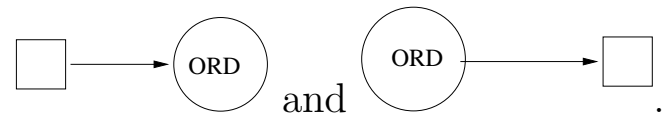

Every word should have a word graph, but not every subgraph of the mind graph needs to have a word associated with it. In this case there are words. In English they are "from" respectively "to". In Japanese we find [6] that KARA respectively E are postpositions with basically the same meaning.

The interesting remarks in the book of Henderson are, quote," E indicates that the substantive it follows is a limit towards which action takes place. The limit is always conceived as fixed, and the action as or like movement in space. If one is thinking primarily of the motion, E is always used. If one is thinking primarily of the fixed limit the present 
tendency is to replace E by NI". NI is described as, quote; "a particle (postposition) which is used to connect two concepts. The first concept, whatever its form, is always thought of as a substantive. The second concept may be in the form of a noun, a verb, or even an entire sentence. The function of NI in any particular sentence depends on the nature of the concepts connected, and therefore cannot be determined until both concpts are known ". For KARA we read, quote; "A postposition, often used as a conjunction. KARA marks what it follows as a limit or base from which action takes place. It may follow: 1.substantives, 2.statements, 3.certain verbs."

It is clear that the ORD-link is at the heart of word graphs for these three Japanese words. "Tookyoo e ikimasu", literally says "Tokyo to go", where ikimasu is a form of "iku" = "go", which will not be discussed.

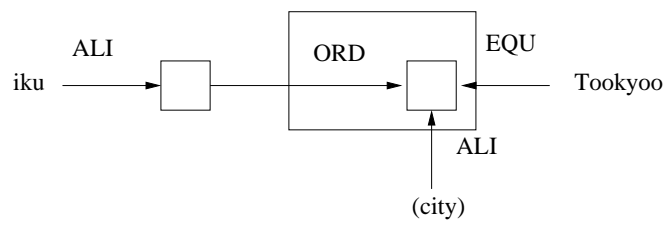

is the basic sentence graph, where the rectangle, in first instance, gives the frame for E. However, it is only the combination with the verb "iku", that contains the "movement in space" as part of its meaning! So a more elaborate treatment should be given. Here the definition of "go" comes into play. "Changing location" may be modeled as "from location at some time to location at later time":

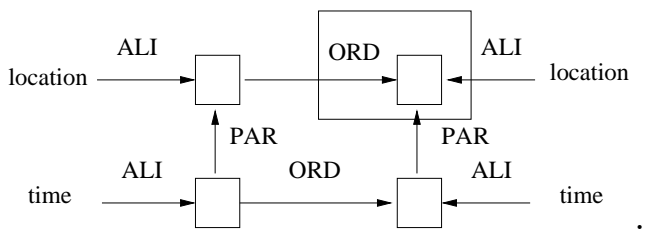

This subgraph will be present in a word graph for "go". The rectangle does not completely determine the meaning of E. As for all words, the meaning is determined by the knowledge graph to which it is thought to belong. If the subgraph occurs in what follows the postposition the choice of $\mathrm{E}$ is justified. With other surrounding knowledge graph structure for the ORD-link the choice is not correct.

Of the many examples for NI given in [6], I chose "oya ni niru", "parent to resemble". The use of "to" in English seems strange but if "resemble" is replaced by "be similar to" the use is justified. "Be similar to" is semantically essentially different from "go to". Also in "otona ni naru", "adult to become", "to" is justified by remarking that "become" can be replaced by "turn into".

Finally, as a third example, in " "A" wa "B" ni hitoshii", " "A" as for "B" to equal", we meet two interesting things. Next to the particle WA, on which later, we see a very clean form of ordering expressed by NI. The semantic content is 


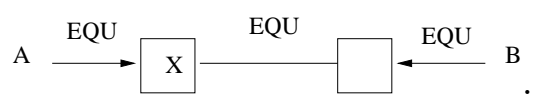

The cross indicates that the focus is on "A". Seen from there the equality is "to "B" ", or " "B" ni", as Japanese would put it.

About KARA we read, quote; "In the early language KARA seems to have been a noun meaning something like "starting point", "origin", and hence "cause" ". The word graph that we proposed, "something from which", could not be described better! Also the link with "cause" is remarkable. After a substantive like "nagai ryokoo", "long journey" KARA expresses "from". After statements KARA expresses "so". After certain verb forms KARA expresses "after", like in "Nippon e mairimashite kara ...", "Japan to arrive after".

We see that, like in English, words show abundant homonymy. In different settings, sentence graphs, the meanings of the same word are different. This strongly underlines the statement that the meaning of a word is the knowledge graph to which it belongs.

We already met the particle WA and saw that is has the function of focusing on something, e.g. in the sentence " "A" wa "B" ni hitoshii". In [6] we read, quote; "A postposition. According to Japanese grammarians its original function was "hai-ta-teki", "exclusive". That is, it set what is followed apart from the other ideas. ... Furthermore, WA not only sets what it follows apart from other ideas, but in doing so suggests that there is in the user's mind some thought of comparison with ideas in the same class as the idea that has been mentioned.".

Consider the following two sentences.

1."Watakushi wa iku ga ano hito wa ikanai", "I as for go but that man as for go not".

2." Asa wa iku ga yoru wa ikanai", "Morning as for go but evening as for go not".

The clearer translations are; "I (will) go, but that man (will) not go.", respectively "Mornings (I will) go, but evenings (I will) not go". Note that the word "ga" is used as a conjunctive, and translated by "but", and differs from the particle GA that we will discuss soon.

The postposition MO is used to indicate that whatever it follows is an addition to other things, [6]. Quote; "If these "other things" have not been previously referred to, MO normally suggests an addition to "other things of the same general kind" and emphasizes a comparison with them. English words for expressing the meaning of MO may be "also", "too" or "even".". The word can be used after substantives, after verbs and adjectives and after adverbs, so after the three main word classes that we distinguished. A very simple example is : "Pen ga aru. Empitsu ga aru. Fude mo aru", "Pen do be. Pencil do be. Brushes even/also be". Here the particle GA is translated by "do". The word graph for MO must have great similarity with that for "and", which is

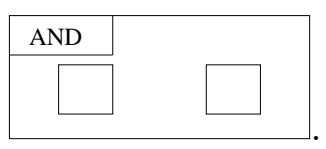


On top of the frame taking two, or more, tokens together, the similarity between the tokens should be represented, which can be done by an ALI-link, so that for MO we can give the word graph

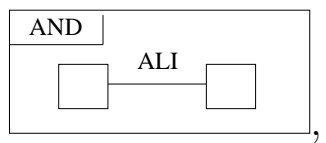

which can be read as "something and something alike".

Kortlandt [11] rightly gives:

$\mathrm{X}$ wa $=$ for an entity $\mathrm{X}$ there is a situation $\mathrm{E}$ in which $\mathrm{X}$ occupies a position.

$\mathrm{X} m o=$ for an entity $\mathrm{X}$ there is a situation $\mathrm{E}$ in which $\mathrm{X}$ occupies the same position as an entity Y.

Note the use of the word "position". A situation is represented by a BE-frame around a knowledge graph, that describes "what the matter is". Within that graph the "entities" have a position.

We now come to the pair GA and WO.

Kortlandt gives:

$\mathrm{X}$ ga $=$ for an entity $\mathrm{X}$ there is a predicate $\mathrm{P}$ of which $\mathrm{X}$ is the subject.

$\mathrm{X}$ wo $=$ for an entity $\mathrm{X}$ there is a predicate $\mathrm{P}$ of which $\mathrm{X}$ is the complement.

Henderson [6] mentions the marking of a noun as subject respectively object as main use too. Recalling our discussion of subject/object versus agent/patient, the word graphs for GA and WO should contain SKO-links as these are used to represent the syntactic function of nouns.

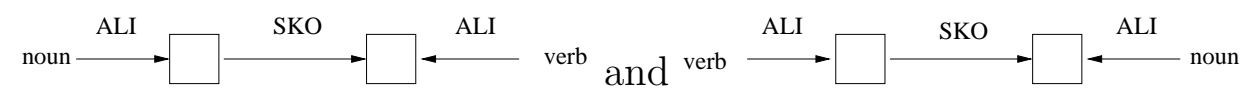

for GA respectively WO would be the result. However, these are parts of the syntactic word graphs of verb and noun, see Zhang [17]. Is it necessary to mention this context? Henderson gives the example "Kanaoka ga uma", "Kanaoka do horse", i.e., in English, "A horse (painted) by Kanaoka". So here two substantives are joined by GA. The relationship between "Kanaoka" and "uma" that is indicated is just that the "horse" is informationally dependent on "Kanaoka", the painter. Hence GA basically just has the SKO-link as its word graph. A similar thing can be said about the particle WO. Like for "from" and "to" and the ORD-arc, we may make a distinction between the word graphs for GA and WO by considering the total graph of the SKO-arc and its subgraphs

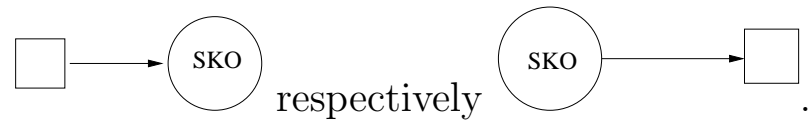

Note that the SKO-link is only used in syntactic word graphs to link a noun and a verb in English. 
As a last remark on GA we quote Henderson: "GA may indicate a psychological subject which is not necessarily the strict grammatical subject. An example is "Watakushi wa kore ga suke desu", "I as for this do fond of be"." We are reminded of "this is the object of desire", strictly stating that "this" is seen as informationally dependent on the "desire".

We now come back to the merological relationships, by considering NA and NO. Again considering the total graph form we can consider the word graphs

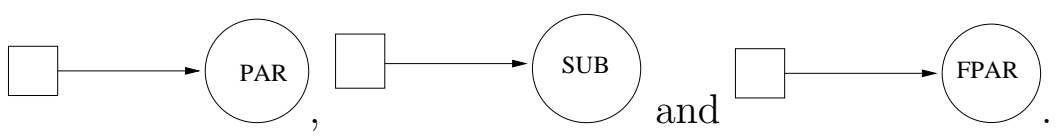

All three are word graph for the English word "with", of which the three meanings, word graphs, are homonyms. Similarly the word graphs

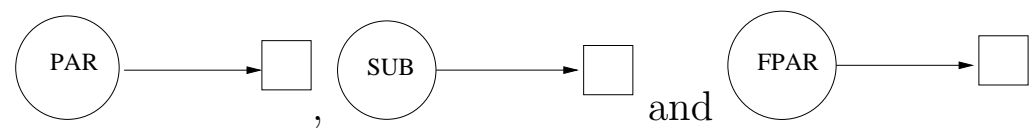

are homonyms of the word "of". This homonymy is the cause of much confusion about the different merological relationships. Note that we essentially consider demi-arcs. We might also use

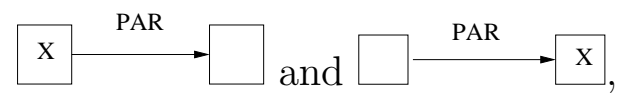

with emphasis on one of the tokens.

Henderson states the following about the postpositional NO, quote; "This NO is a particle (postposition) used to join two substantives. Its function is to indicate that the first substantive in some way characterizes the second."

Kortlandt states:

"X no = for an entity $\mathrm{X}$ there is an entity $\mathrm{Y}$ which carries the features of $\mathrm{X}$ indirectly".

I.e. the features may exist without the entity.

The word "characterizes" used by Henderson is somewhat unfortunate as it had better be reserved for parts of definitions. We will consider some examples that differ in the semantics of NO from the point of view of knowledge graph ontology.

"Anata no hon", "You of book". Here the possession is expressed, typically a PAR-link from "anata" to "hon".

"Kin no yubiowa", "Gold of ring". Here we meet an FPAR-link in disguise. A ring is, by definition, material. Gold instantiates, via an EQU-arc that material.

"Kodomo no kao", "Child of face". Here we would choose the SUB-link in the word graph.

Like for "of", no distinction is made in the use of NO. The context determines the more detailed semantics. We quote from Henderson; "There will usually be very little difficulty in determining from the context what kind of characterization is implied, though 
unfortunately NO is such a convenient word that there is a natural tendency to overwork it, rather than go to the trouble of accurate definition." The description given by Kortlandt is therefore somewhat restrictive, when stressing a relationship between entities. Henderson gives: "anata kara no tegami", "you from of letter", where "you from" is not what we would call an "entity". The wider range of possible use of NO also comes forward in "akai no ga kimi no de, shiroi no ga boku no da", "red thing do you of be, white thing do I of be", or "the red one is yours and the white one is mine".

Several remarks are due here. The NO after "akai" and "shiroi" is the substantial "no", probably stemming from "mono", "thing". The NO after "kimi" and "boku" is expressing the genitive, here for a PAR-link. The substantivation is essentially different from the part-of relationship, here used without another entity.

Henderson states: "NA is a particle with a large number of uses.".

Kortlandt gives as description:

$\mathrm{X}$ na $=$ for an entity $\mathrm{X}$ there is an entity $\mathrm{Y}$ which carries the features of $\mathrm{X}$ directly. I.e., the features do not exist without the entity.

Henderson states: "NA may be the equivalent of the modern NO. This is a definitely archaic use of NA." and "A glossary gives "genki na", "high-spirited, vigorous".

Kortlandt gives an example of Takeuchi; "genki na okaasan", "high-spirited mother". "Genki na" indeed seems to be an archaic standard combination. "Heiwa na kuni", "peace of land", i.e., "peaceful country" is another example of the use of NA in a merological sense. Neither "genki" nor "heiwa" are features that have to exist with respectively "okaasan" and "kuni". So the difference made by Kortlandt between NO and NA, by distinguishing direct and indirect features, seems doubtful.

We still have to discuss the three word graphs for "with". For this we have to consider another particle, namely DE. Henderson states, quote; "As a postposition, it normally indicates that the substantive it follows is used in the performance of an action." "Pen de tegami wo kaku", "Pen with letter object write". The sentence graph would be:

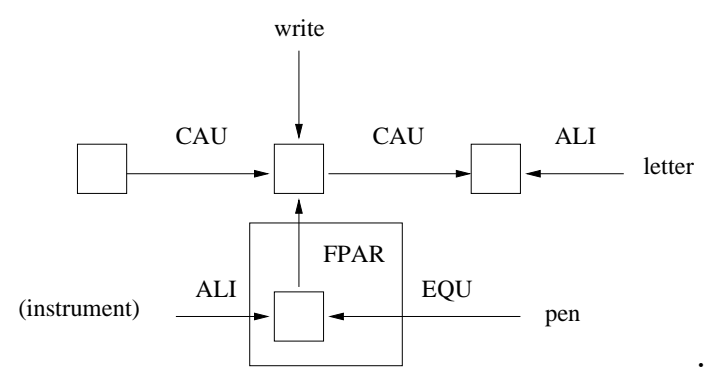

Here the pen is seen as instantiation of an instrument that is a property of writing, hence the FPAR-link. The subgraph indicated by the rectangle is the word graph for DE. If some action A takes place in a garden, "niwa", one may say "niwa de A". Here DE is usually translated as "in" or "at". This can be understood by expanding the action A. An action takes place at some location, that has a PAR-link with the verb describing the action A. In the example "niwa" instantiates the location and DE corresponds with the subgraph 


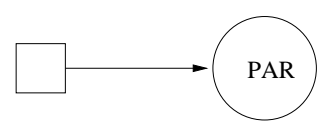

from location to verb.

We see that the particles that are used most often indeed correspond to elements of the knowledge graph ontology and "glue" the words of the main classes of words, verbs, nouns and adwords, together. However, there are more particles!

\section{Further particles}

We have discussed the most common particles. However, there are considerably more. We have met particles the word graphs of which contained the SUB-link, the PAR-link, the FPAR-link, the ORD-link and the SKO-link, all five oriented links, not any particles with the symmetric EQU-, ALI- or DIS-link, except for MO. Nor did we encounter the CAU-link in this sense, again casting some doubts on the basic nature of this type of link.

To choose some further particles, it is interesting to visit the internet, where many sites give an account of Japanese particles. I chose Ken Butler's site [4]. He mentions WA, NO, WO, MO, GA, but not NA! Next to these he mentions KA, NI, DE, E, NE/YO, TO, KARA/MADE, YORI, HODO, DAKE, SIKA, GURAI, BAKARI, DEMO, SAE and TOMO. We will discuss each of these shortly to see what makes them different from the first five, already discussed.

KA corresponds to the question mark and, like that, is connected by a PAR-link to the whole sentence graph it follows.

NI is used to connect two concepts. The first is always thought of as a substantive. Henderson states, quote; "Dictionaries often translate NI as meaning "at", "on", "in", "to", "into", "for", "of", "with", "from", "as", "by", etc. Most students find it easier to think of it as meaning simply "in connection with"." If this is so then any type of relationship from the knowledge graph ontology may play a role in the many word graphs of homonyms of NI that would represent the various interpretations.

An important example is "Tookyoo ni", "in Tokyo", or "soko ni", "there(by you)", where "soko" is "that place where you are". Whatever is "in Tokyo" has a location that is included in the area of Tokyo, and hence NI has a corresponding SUB-link. We refer to Hoede and $\mathrm{Li}$ [8] for a related discussion of the many words in Chinese for "in". In Chinese the context is included in the word meaning in 15 different ways. Here, in Japanese, the context does not have an effect on the word chosen, a remarkable difference.

In "sono toki ni", "that time at", we encounter an instantiation of a time. That time is represented by a token of type time linked to some verb by a PAR-link. In "kore wo anata ni agemasu", "this (object) you to present", it seems that an ORD-link is involved. In "oya ni niru", "parent "ni" resemble", the English word is difficult to give as "resemble" means "being similar to". So again an ORD-link seems to be captured by the use of NI. 
We already considered "" A wa "B" ni hitoshii", " "A" as for "B" to equal". The use of NI is like in the former example. By the focus on "A" by WA, the EQU-link becomes oriented towards "B".

DE was already discussed, as was E.

Here we should point out the connection of E with MADE. That postposition, quote;" has the force of the English "upto". That is, it marks what it follows a limit "up to" (and including) which an action takes place." Note that E does not have the extra meaning that "upto" has with respect to "to".

Of the remaining particles the foremost one is TO. Henderson states, quote; " TO is a connective particle, with an additional reflexive force, somewhat like "that" or "that way". It indicates a union in all its uses.". We only quote two examples. "Amari nagai to oreru". "Too much long that way break", or "Break it off when it is too long". "Tokyoo to Oosaka to Kobe to ni yooji", " Tokyo and Osaka and Kobe in business", or " Business in Tokyo, Osaka and Kobe".

In a way NI and TO are the most typical particles, used to express a variety of uses. The type of relationship expressed depends on the linguistic units that they connect. As is to be expected, both particles are combined with other words in order to be more specific. Henderson mentions : ni mo, ni naru, ni oite, ni okaserarete, ni okeru, ni shite, ni shite mo, ni shite wa, nite, ni totte, ni wa, ni yoru. Next to TO he mentions to iu, to ka, to mo, to shite, to shite mo, to shite wa, to su, tote, tote wa, to wa, to zo.

$\mathrm{NE}$ and YO are, like KA, attached to complete sentences meaning "isn't it?" respectively "!".

YORI marks what it follows as a limit or base from which some action takes place, or beyond which some state exists, usually translated by "from". This is similar to MADE versus E. YORI is like KARA but with an extra instantiation aspect.

BAKARI, DAKE, HODO and KURAI are in a sense related in that they express special aspects of a standard or measurement, like "about", "approximately", "exactly" or "just".

DEMO is a logic word meaning "although" or "even if". SIKA means "only". SAE means " to that extent". TOMO expresses something like "and ... and ...".

\section{Conclusion.}

It seems clear that particles correspond to the particular ways in which knowledge graph links are thought to connect tokens, i.e. to certain subgraphs of the mind graph. This is in line with the idea that thinking is linking somethings. In this way Japanese strongly supports knowledge graph theory, in particular the other idea that the structure is the meaning.

\section{Acknowledgement}

The author would like to thank Professor Kortlandt for his stimulating suggestions. 


\section{References}

[1] Bakker, R.R., Knowledge Graphs : Representation and Structuring of Scientific Knowledge, $\mathrm{PhD}$ thesis, University of Twente, Enschede, The Netherlands, ISBN 909001963-4, (1987).

[2] Berg, H. van den, Knowledge Graphs and Logic : One of Two Kinds, PhD thesis, University of twente, Enschede, The Netherlands, ISBN 90-3651835-0, (1994).

[3] Bondy J.A. and U.S.R. Murty, Graph Theory with Applications, The MacMillan Press, London and Basingstoke, SBN 333-17791-6, (1976).

[4] Butler, K., A Guide to the Functions of Structural Particles in Japanese, http://home.inter.net/kenbutler/particlehome.html, (2003).

[5] Ebeling, C.L., Een inleiding tot de syntaxis, Summary (J.Wiedenhof en R.Smeets, eds.), The Hakuchi Press, Leiden, ISBN 90-711760-8, (1994).

[6] Henderson, H.G., Handbook of Japanese Grammar, Allen \& Unwin , London, (1945).

[7] Hoede, C., Syntax and semantics: A comparison of the structuralistic language theory of Ebeling with knowledge graph theory, Memorandum No. 1710, Faculty of Mathematical Sciences, University of twente, Enschede, The Netherlands, ISSN 0169-2690, (2004).

[8] Hoede, C. and X.Li, Word Graphs: The First Set; In: Conceptual Structures. Knowledge Representation as Interlingua, Aux. Proc. of the Fourth International Conference on Conceptual Structures, (P.W.Eklund, G.Ellis and G.Mann, eds.), Bondi beach, Sydney, Australia, 81-93, (1996).

[9] Hoede, C. and X. Liu, Word Graphs: The Second Set; In: Conceptual Structures: Theory, Tools and Applications, Proc. of the 6th International Conference on Conceptual Structures, (M-L. Mugnier and M. Chein, eds.), Montpellier, Springer Lecture Notes in Artificial Intelligence no. 1453, 375-389, (1998).

[10] Hoede, C. and L. Zhang, Word Graphs: The Third Set; In: Conceptual Structures: Broadening the Base, Proc. of the 9th International Conference on Conceptual Structures, (H.S. Delugach and G. Stumme, eds.), CA, USA, Lecture Notes in Artificial Intelligence no. 2010, 15-28, (2001).

[11] Kortlandt, F., Japanese wa, mo, ga, wo, na, no, http://www.kortlandt.nl/publications/, (2002).

[12] Peirce, C.S., On the Algebra of Logic. American Journal of Mathematics, 7, 180-202, (1885). 
[13] Russell, B., History of Western Philosophy, Book Three, Chapter XVII, Allen \& Unwin, London, ISBN 0-04-109009-8, (1961).

[14] Smit, H.J., Consistency and Robustness of Knowledge Graphs, PhD thesis, University of Twente, Enschede, The Netherlands, ISBN , (1991).

[15] Vries, P.H. de, Representation of Science Texts in Knowledge Graphs, PhD thesis, University of Groningen, Groningen, The Netherlands, ISBN , (1989).

[16] Willems, M., Chemistry of Language, PhD thesis, University of Twente, Enschede, The Netherlands, ISBN 90-9005672-6, (1993).

[17] Zhang, L., Knowledge Graph Theory and Structural Parsing, PhD thesis, University of Twente, Enschede, The Netherlands, ISBN 90-3651835-0, (2002). 